\title{
3D global simulations of a cosmic-ray-driven dynamo in dwarf galaxies
}

\author{
H. Siejkowski ${ }^{1,2}$, K. Otmianowska-Mazur ${ }^{2}$, M. Soida ${ }^{2}$, D. J. Bomans ${ }^{3,4}$, and M. Hanasz ${ }^{5}$ \\ 1 AGH University of Science and Technology, ACC Cyfronet AGH, ul. Nawojki 11, PO Box 386, 30-950 Kraków 23, Poland \\ e-mail: h.siejkowski@cyfronet.pl \\ 2 Astronomical Observatory of the Jagiellonian University, ul. Orla 171, 30-244 Kraków, Poland \\ 3 Astronomical Institute of Ruhr-University Bochum, Univeristätsstr. 150/NA7, 44780 Bochum, Germany \\ ${ }^{4}$ Ruhr-University Bochum Research Department: Plasmas with Complex Interactions, Univeristätsstr. 150, 44780 Bochum, Germany \\ 5 Centre for Astronomy, Nicolaus Copernicus University, Faculty of Physics, Astronomy and Informatics, Grudziadzka 5, \\ 87100 Toruń, Poland
}

Received 11 September 2012 / Accepted 29 November 2013

\section{ABSTRACT}

\begin{abstract}
Context. Star-forming dwarf galaxies can be seen as the local proxies of the high-redshift building blocks of more massive galaxies according to the current paradigm of the hierarchical galaxy formation. They are low-mass objects, and therefore their rotation speed is very low. Several galaxies are observed to show quite strong magnetic fields. These cases of strong ordered magnetic fields seem to correlate with a high, but not extremely high, star formation rate.

Aims. We investigate whether these magnetic fields could be generated by the cosmic-ray-driven dynamo. The environment of a dwarf galaxy is unfavourable for the large-scale dynamo action because of the very slow rotation that is required to create the regular component of the magnetic field.

Methods. We built a 3D global model of a dwarf galaxy that consists of two gravitational components: the stars and the dark-matter halo described by the purely phenomenological profile proposed previously. We solved a system of magnetohydrodynamic equations that include an additional cosmic-ray component described by the fluid approximation.

Results. We found that the cosmic-ray-driven dynamo can amplify the magnetic field with an exponential growth rate. The $e$-folding time is correlated with the initial rotation speed. The final mean value of the azimuthal flux for our models is on the order of few $\mu \mathrm{G}$ and the system reaches its equipartition level. The results indicate that the cosmic-ray-driven dynamo is a process that can explain the magnetic fields in dwarf galaxies.
\end{abstract}

Key words. magnetohydrodynamics (MHD) - dynamo - galaxies: magnetic fields - galaxies: dwarf - methods: numerical

\section{Introduction}

Star-forming dwarf galaxies are smaller, fainter, and less massive than their spiral counterparts, but they are the most numerous population in the Universe (Blanton et al. 2002). The magnetic fields in dwarf galaxies may play a very important role. First, the observations show that the magnetic field is an important source of pressure for the interstellar medium (Boulares \& Cox 1990). It is often assumed that the interstellar medium (ISM) in galaxies is in equipartition with the almost equally distributed energy in magnetic fields, cosmic rays, and turbulence. Second, these objects are less massive, hence the gravitational potential well is shallower and this facilitates the escape of the gas from the galaxy in the form of galactic winds (Mac Low \& Ferrara 1999). This wind can also drag the magnetic field out of the disk into the intergalactic medium (IGM) since the magnetic field is frozen into the outflowing plasma. Possible magnetisation of the IGM via the magnetised wind has been studied by Bertone et al. (2006) and for dwarf galaxies by Kronberg et al. (1999) and Scannapieco \& Brüggen (2010). Local simulations of the cosmic-ray dynamo process in dwarf galaxies (Siejkowski et al. 2010) show significant loss of the magnetic field from the domain and it depends on the supernova rate (SNR). Studies of dwarf galaxy formation by Dubois \& Teyssier (2010) also implied IGM seeding via galactic winds. Additionally, there are studies on galactic winds driven by cosmic rays by Booth et al. (2013) and Hanasz et al. (2013).

Strong magnetic fields were discovered in a bright dwarf irregular galaxy NGC 4449 with a total field strength of about $12 \mu \mathrm{G}$ and a regular component of up to $8 \mu \mathrm{G}$ (Klein et al. 1996; Chyży et al. 2000). Kepley et al. (2010) reported similar magnetic fields in NGC 1569 . The radio observations of these two galaxies show some large-scale magnetic fields with a sign of a spiral pattern, but no optical counterparts. The rotation measure maps imply that the magnetic field is almost parallel to the disk plane. In other dwarf objects such as NGC 6822, IC 10 (Chyży et al. 2003) and the Large Magellanic Cloud (Klein et al. 1989; Gaensler et al. 2005) the observed magnetic fields are weaker, reaching a value about 5-7 $\mu \mathrm{G}$. It is worth noting that these galaxies, especially NGC 1569 and NGC 4449, are under strong influence of infalling gas from the surroundings, which have a significant impact on the magnetic-field structure.

Observations of the above mentioned objects brought some insight into the dynamo process in dwarf galaxies, but all these objects are optically bright and showed disturbed kinematics. Therefore the sample might be influenced by strong selection effects. Chyży et al. (2011) completed a sample of dwarf and small irregular galaxies from the Local Group. They found that the star formation rate (SFR) regulates the strength of magnetic fields, because the SNR, which is proportional to the SFR, contributes 
to drive the turbulence in the ISM. These results are very similar to the conclusions of our previous theoretical study (Siejkowski et al. 2010) of the cosmic-ray-driven dynamo in the medium of irregular galaxies. Chyży et al. (2011) have also investigated a possible relation between the maximum $v_{\text {rot }}$ and the magneticfield strength. For slow rotation $\left(<40 \mathrm{~km} \mathrm{~s}^{-1}\right)$ all galaxies have weak fields, below $4 \mu \mathrm{G}$. With increasing maximum rotation speed in the following objects the observed magnetic fields are stronger. However, some objects, such as NGC 4449, show very strong magnetic fields, but their rotation is rather slow. The relation of the magnetic field versus the maximum rotation speed is probably distorted by the fact that objects with strong magnetic fields are undergoing heavy star formation. The contribution to the turbulent energy by supernovae explosions can cause strong disturbances in the velocity pattern, therefore estimating the maximum rotation speed and assigning a clear rotation curve is difficult.

It is believed that the magnetic dynamo is responsible for the strength and structure of magnetic fields in galaxies (Beck 2009). One of the recent dynamo models is driven by thermal energy output from supernovae explosions, described by Gressel et al. (2008a,b). These authors found that the $e$-folding time of the amplification mechanism is about $\tau_{e}=250 \mathrm{Myr}$ and is dependent on the rotation speed. Another model suggested by Parker (1992) relies on the buoyancy instability of interstellar medium filled with magnetic fields and cosmic rays (Parker 1965). The cosmic-ray-driven dynamo was constructed for the first time within the framework of a local shearing box model by Hanasz et al. (2004). An extensive parameter study of this local model was presented in Hanasz et al. (2009a). The $e$-folding time-scale of the magnetic-field amplification by the cosmic-ray-driven dynamo is generally comparable to the galactic rotation period and can be as short as $140 \mathrm{Myr}$ (Hanasz et al. 2006). Siejkowski et al. (2010) investigated the cosmic-ray driven dynamo action in lowmass objects, such as dwarf- and intermediate-mass irregular galaxies. This study led to the following results: the growth rate of the magnetic field is strongly dependent on the rotation speed, but for objects with $v_{\text {rot }}>40 \mathrm{~km} \mathrm{~s}^{-1}$ the saturation of the dynamo is reached after the same period of time. The $e$-folding time is also dependent on the SNR and the time of quiescent state (no supernovae activity). The larger the SNR, the faster the growth rate, but excessive supernova activity can suppress the dynamo action. Hanasz et al. (2009b) demonstrated cosmic-ray-driven dynamo action via global disk spiral galaxies, and Kulpa-Dybeł et al. (2011) confirmed the result for barred galaxies. They found that the azimuthal magnetic flux grows on a time-scale of about $270 \mathrm{Myr}$.

\section{Numerical model and setup}

To simulate the dwarf galaxy model a numerical code called Godunov-MHD was employed (Kowal et al. 2009). It solves the system of ideal magnetohydrodynamic (MHD) equations in a conservative form in 3D space. The key elements and assumptions of the cosmic-ray-driven dynamo global galactic model were adopted from Hanasz et al. (2009b). Below we describe our choice of simulation parameters. An isothermal equation of state was assumed, that is $p \equiv \rho c_{\mathrm{s}}^{2}$, where $c_{\mathrm{s}}$ is the isothermal speed of sound set to $7 \mathrm{~km} \mathrm{~s}^{-1}$, which corresponds to a gas temperature of $6000 \mathrm{~K}$. We assumed the magnetic diffusivity $\eta$ to be constant and equal to $0.1 \mathrm{kpc}^{2} \mathrm{Gyr}^{-1}=3 \times 10^{25} \mathrm{~cm}^{2} \mathrm{~s}^{-1}$. The investigation by Hanasz et al. (2009a) showed that this value is optimal for the growth of the magnetic field in the buoyancydriven dynamo.
The cosmic ray (CR) component is described by the diffusion-advection transport equation in terms of fluid approximation following Schlickeiser \& Lerche (1985) and Hanasz \& Lesch (2003). We related the CR pressure to the CR energy density $e_{\mathrm{cr}}$ via the adiabatic CR index, that is $p_{\mathrm{cr}} \equiv\left(\gamma_{\mathrm{cr}}-1\right) e_{\mathrm{cr}}$ and $\gamma_{\mathrm{cr}}=14 / 9$ adopted from Ryu et al. (2003). Following Giacalone \& Jokipii (1999), we assumed that the diffusion of cosmic rays is anisotropic with respect to the direction of the magnetic field. The typical values of the diffusion coefficient found by modelling CR data (see e.g. Strong et al. 2007) are $(3 \div 5) \times 10^{28} \mathrm{~cm}^{2} \mathrm{~s}^{-1}$. The applied value of the perpendicular $\mathrm{CR}$ diffusion coefficient is $K_{\perp}=1 \mathrm{kpc}^{2} \mathrm{Gyr}^{-1}=3 \times 10^{26} \mathrm{~cm}^{2} \mathrm{~s}^{-1}$ and the parallel one is $K_{\|}=10 \mathrm{kpc}^{2} \mathrm{Myr}^{-1}=3 \times 10^{27} \mathrm{~cm}^{2} \mathrm{~s}^{-1}$. The parallel diffusion coefficient is $10 \%$ of the realistic value because the time-step of the explicit diffusion algorithm becomes prohibitively short when the diffusion coefficient is too high. The effect of the reduced CR diffusion coefficients was investigated by Hanasz et al. (2009a), showing that the magnetic-field growth only slightly depends on the $K_{\|}$value, but the key factor in the cosmic-ray-driven dynamo is the anisotropy of the diffusion.

A single supernova explosion was modelled by a 3D Gaussian distribution of cosmic-ray energy input and equals $10 \%$ of the canonical kinetic energy output of the supernova explosion, that is $10^{51} \mathrm{erg}$. In the initial period in $t \in$ (100 Myr, $400 \mathrm{Myr}$ ), every one in ten explosions injects a randomly oriented dipole magnetic field into the ISM in addition to the CR energy input. The injection of the magnetic field is only to seed the dynamo action through a random field at the beginning of the simulation. We stopped the injection because (1) it allowed us to study the efficiency of the pure cosmic-ray-driven dynamo while the injected magnetic field was only the seed field and (2) after several hundred Myr the seeding becomes insignificant with respect to the existing field.

The position of each supernova explosion was chosen randomly with respect to the local gas density, that is $\rho^{3 / 2}$. This follows the simple self-gravitational picture drawn by Schmidt (1959) and Kennicutt (1989), where the large-scale star formation rate volume density scales with $\rho^{3 / 2}$. The number of supernovae is given by the supernova explosion frequency over the disk area. In our model its value was set to $f_{\mathrm{SN}}=3 \times$ $10^{3} \mathrm{kpc}^{-2} \mathrm{Gyr}^{-1}$ and it was constant during whole simulation time. This value corresponds roughly to the density of the star formation rate ( $\Sigma \mathrm{SFR}$ ) equal to $10^{-3} M_{\odot} \mathrm{yr}^{-1} \mathrm{kpc}^{-2}$ and is a typically found in non-starbursting dwarf galaxies (see e.g. Chyży et al. 2011).

The dwarf galaxy potential well is given by two components: a dark-matter (DM) halo and a thin stellar disk. This type of galaxy has no bulge (Governato et al. 2010), which is present in more massive disk galaxies. In our model the stars are distributed in an infinitesimally thin Kuzmin disk following previous numerical works on dwarf galaxies (Marcolini et al. 2003, 2004). For the DM halo we used the purely phenomenological profile proposed by Burkert (1995). Its gravitational potential is described by

$$
\begin{aligned}
\phi_{\mathrm{DM}}(r)= & -\pi G \rho_{0} r_{0}^{2}\left\{\pi-2\left(1+x^{-1}\right) \arctan x\right. \\
& \left.+2\left(1+x^{-1}\right) \ln (1+x)-\left(1-x^{-1}\right) \ln \left(1+x^{2}\right)\right\},
\end{aligned}
$$

where $G$ is the gravitational constant, $\rho_{0}$ is the central density, $r_{0}$ is the core radius, $r$ is the distance to the centre, and $x \equiv r / r_{0}$. 
H. Siejkowski et al.: 3D global simulations of a cosmic-ray-driven dynamo in dwarf galaxies

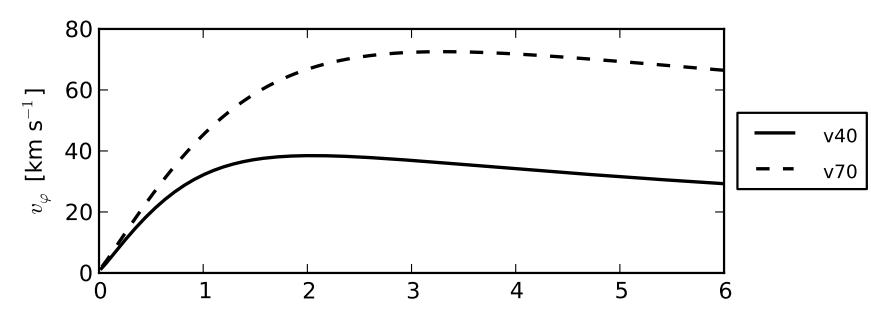

Fig. 1. Rotation curves for models from Table 1.

Table 1. Parameters of the models v40 and v70.

\begin{tabular}{lccc}
\hline \hline Parameter & v40 & v70 & Unit \\
\hline Mass of stars & 1.0 & 6.0 & $10^{9} M_{\odot}$ \\
$\rho_{0}$ & 21.5 & 15.0 & $10^{6} M_{\odot} \mathrm{kpc}^{-3}$ \\
$r_{0}$ & 1.4 & 2.4 & $\mathrm{kpc}$ \\
$\rho_{\mathrm{g}}$ & 29.5 & 29.5 & $10^{6} M_{\odot} \mathrm{kpc}^{-3}$ \\
$R_{\mathrm{c}}$ & 1.2 & 3.6 & $\mathrm{kpc}$ \\
$v_{\varphi}^{\max }$ & 40 & 70 & $\mathrm{~km} \mathrm{~s}^{-1}$ \\
\hline
\end{tabular}

The gas distribution of a dwarf galaxy was set in hydrostatic equilibrium in its initial state. The gas density distribution in equatorial plane was assumed in the following form:

$\rho(R, z=0)=\frac{\rho_{\mathrm{g}}}{\left[1+\left(R / R_{\mathrm{c}}\right)^{2}\right]^{2}}$,

where $\rho_{\mathrm{g}}$ and $R_{\mathrm{C}}$ are the central gas density and core radius, respectively. To find the global gas distribution we used the potential method described in Wang et al. (2010). The detailed parameters of the gravitational potentials are shown in Table 1 and its values were mostly taken from Marcolini et al. (2003) with only slight modifications. From the gas distribution the CR component distribution was found, assuming that they are in pressure equilibrium. We assumed $B=0$ at $t=0$.

The dwarf galaxy model was simulated in 3D Cartesian domain of size $14 \times 14 \times 7 \mathrm{kpc}$ in $x, y, z$ coordinates, respectively. The cell size was $50 \mathrm{pc}$ in each direction. Upper and lower boundary conditions were set to outflow, and we enforced the gas to follow the prescribed rotation curve in the horizontal domain boundaries.

\section{Results and discussion}

The models presented in Table 1 correspond to the different maximum rotation speeds. We set up models with $v_{\varphi}^{\max }=40 \mathrm{~km} \mathrm{~s}^{-1}$ and $70 \mathrm{~km} \mathrm{~s}^{-1}$. The model v40 corresponds to objects like NGC 1569 or IC 10, while the v70 model mimics the Large Magellanic Cloud or NGC 6822 (e.g. Chyży et al. 2011). The rotation curves of these models are shown in Fig. 1.

The evolution of the total magnetic energy and the magnetic flux are shown in Fig. 2. In both models the magnetic-field energy and the magnetic flux are growing exponentially in time. The initial peak, visible up to $t=400 \mathrm{Myr}$, is caused by the magnetic field injected into the system via the magnetised supernovae. Later the magnetic field injection was stopped, and the magnetic growth was only caused by the operation of the dynamo.

After the initial injection of the magnetic field the system experiences a period of stabilization and the magnetic-field energy decreases by about half an order of magnitude. After this, at about $t=1 \mathrm{Gyr}$, the magnetic field starts to be amplified. In the evolution of the magnetic flux we see a constant growth, without
Table 2. Comparison of the simulation results with observations.

\begin{tabular}{rccc}
\hline \hline Model & $\begin{array}{c}v_{\phi}^{\max } \\
{\left[\mathrm{km} \mathrm{s}^{-1}\right]}\end{array}$ & $\begin{array}{c}\Sigma S F R \\
{\left[M_{\odot} \mathrm{yr}^{-1} \mathrm{kpc}^{-2}\right]}\end{array}$ & $\begin{array}{c}B \\
{[\mu \mathrm{G}]}\end{array}$ \\
\hline $\mathrm{v} 40$ & 40 & $10^{-3}$ & 1.0 \\
$\mathrm{v} 70$ & 70 & $10^{-3}$ & 8.0 \\
\hline Object & & & \\
\hline IC 1613 & 37 & $3.7 \times 10^{-4}$ & $2.8 \pm 0.7$ \\
NGC 4449 & 40 & $1.7 \times 10^{-2}$ & $9.3 \pm 2.0$ \\
NGC 1569 & 42 & $1.5 \times 10^{-1}$ & $14.0 \pm 3.0$ \\
IC 10 & 47 & $5.2 \times 10^{-2}$ & $9.7 \pm 2.0$ \\
NGC 6822 & 60 & $6.0 \times 10^{-3}$ & $4.0 \pm 1.0$ \\
LMC & 72 & $4.0 \times 10^{-3}$ & $4.3 \pm 1.0$ \\
\hline
\end{tabular}

Notes. The last column shows the value of mean magnetic field in the disk $(\bar{B})$ for the models; for real objects the total magnetic field $\left(B_{\text {tot }}\right)$ is given. All the observational values are taken from Chyży et al. (2011).

any stabilization periods. This is because the injected magnetic field is random and is ordered by the global rotation. This also suggests that during the stabilization period the randomly oriented magnetic field is expelled and/or dissipated by magnetic diffusivity beyond the simulation domain, while the ordered azimuthal magnetic flux is retained.

When the dynamo begins to operate effectively, the magnetic field and its azimuthal part are exponentially amplified in time. The growth rate of the azimuthal magnetic flux, measured by the $e$-folding time, is different for each of the models and equals 1023 Myr for v40 and 458 Myr for v70. For model v70 the equipartition level is reached at about $t=5 \mathrm{Gyr}$. Around that time some peaks appear in the evolution of the magnetic-field energy, but when the equipartition level is reached - they become damped. In model $\mathrm{v} 40$ the equipartition level is reached at $t=10 \mathrm{Gyr}$, but the transition is smoother than in the previous case. In both cases the saturation of the growth of $B_{\phi}$ occurs at the same time as for the magnetic energy.

The final mean value of the magnetic field for model v40 is $\bar{B}(t=10 \mathrm{Gyr})=1.0 \mu \mathrm{G}$ and for model v70 it is $\bar{B}(t=5 \mathrm{Gyr})=$ $8.0 \mu \mathrm{G}$. The results are compared with the magnetic field found in observations of dwarf galaxies in Table 2 . The model v70 can be compared with NGC 6822 and the Large Magellanic Cloud, which rotate as fast as $60-70 \mathrm{~km} \mathrm{~s}^{-1}$, and whose $\Sigma$ SFR is of the order of $10^{-3} M_{\odot} \mathrm{yr}^{-1} \mathrm{kpc}^{-2}$. In both cases the observed magnetic field is about $4 \mu \mathrm{G}$. The model $\mathrm{v} 40$ with respect to its rotational velocity can be compared with galaxies such as IC 1613, IC 10, NGC 4449, and NGC 1569. The observed magnetic field in the first object is about $2.8 \mu \mathrm{G}$, which agrees well with our simulations. In other objects the magnetic field is much higher, starting from $9 \mu \mathrm{G}$ up to even $14 \mu \mathrm{G}$. However, these objects have a much higher SFR. In our modelling the applied supernova frequency is equivalent to $\Sigma S F R=10^{-3} M_{\odot} \mathrm{yr}^{-1} \mathrm{kpc}^{-2}$, while in these galaxies the observed values are given in Table 2. The $\Sigma$ SFR value is comparable only for IC 1613 , but in other cases the observed values are much higher. This leads to the conclusion that the enhanced SFR can increase the magnetic field in the galaxy disk, but to state it more clearly, more numerical investigations need to be made.

In Fig. 3 the slices through the domain of model v70 are shown for selected time-steps. The evolution of $B_{\phi}$ shows the initially randomly oriented magnetic field, injected through the magnetised supernova explosions, is efficiently regularised. In the $x y$ plane at $t=0.15 \mathrm{Gyr}$ randomly positioned spots appear 

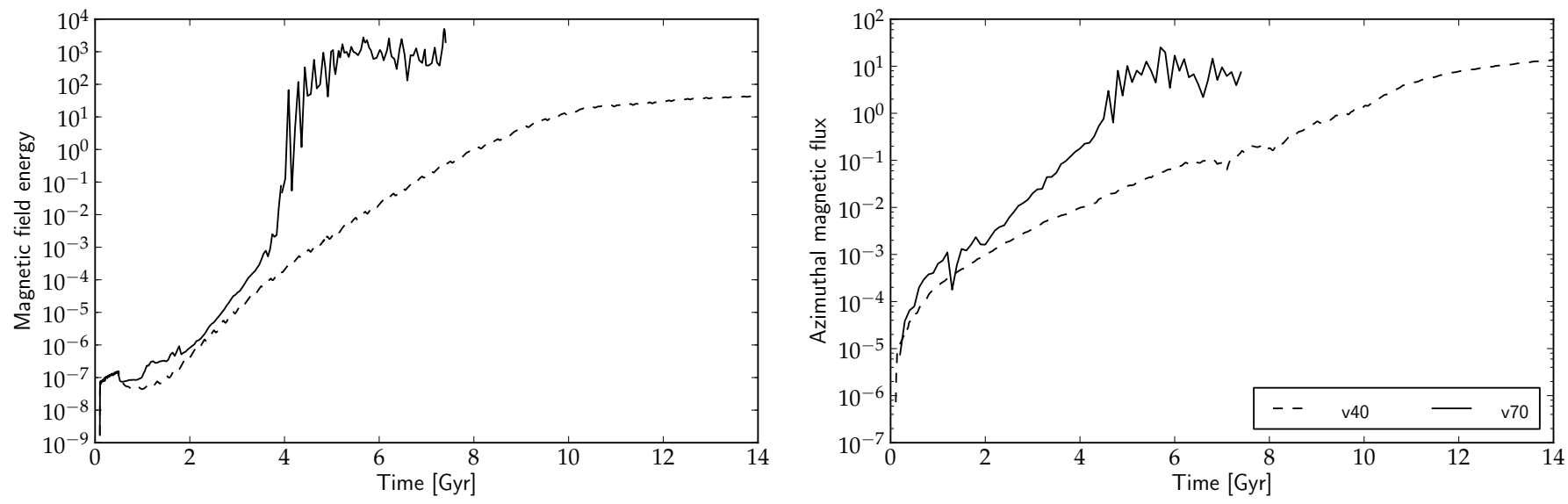

Fig. 2. Left panel: magnetic-field energy evolution for models from Table 1. Right panel: total azimuthal magnetic flux evolution. The plotted value is the absolute value of $B_{\phi}$, because it changes its sign at the beginning of the simulation and therefore it is difficult to show it in a log-plot.
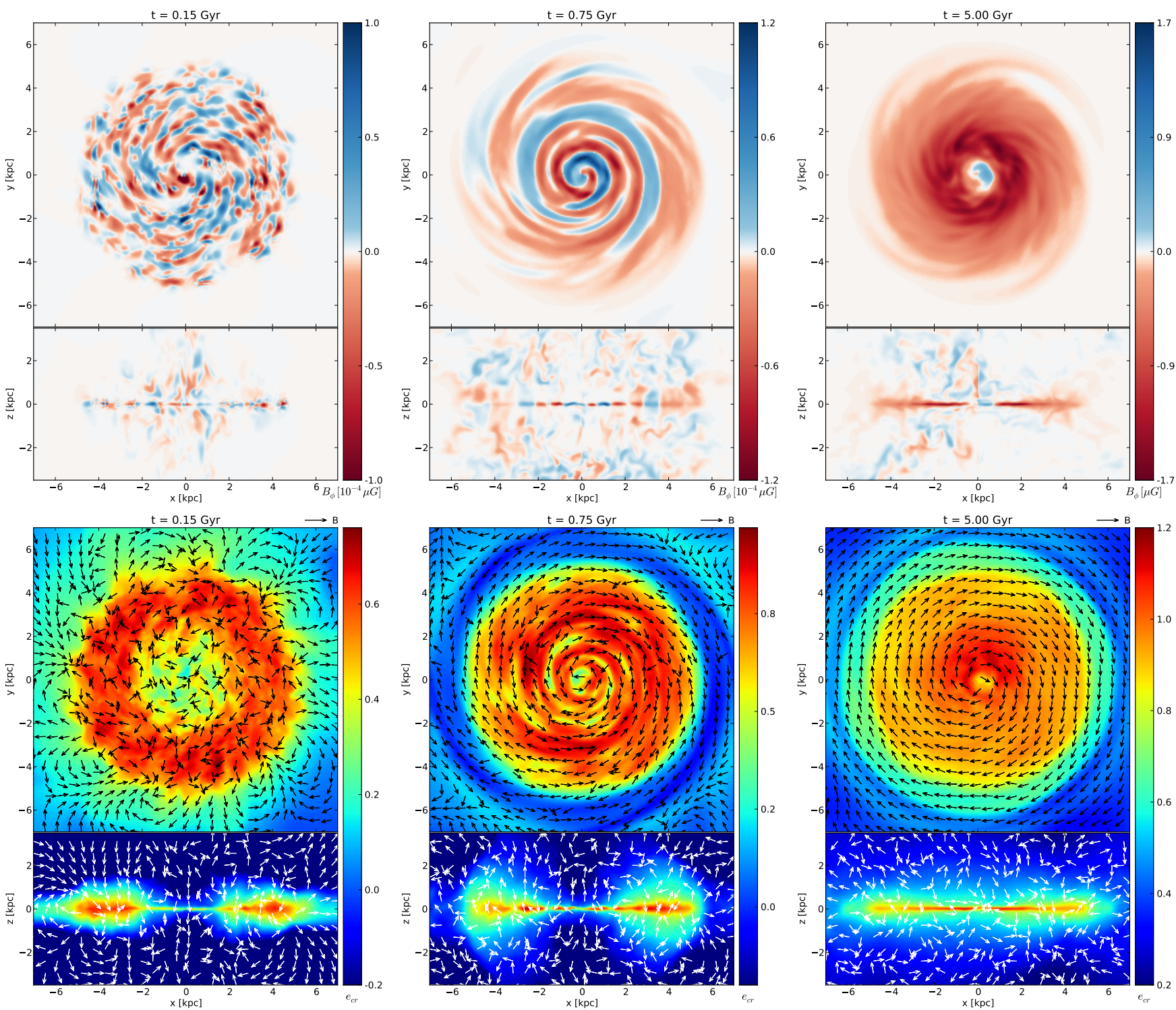

Fig. 3. Evolution of the magnetic field. Upper panels: evolution of the $B_{\phi}$ component (expressed in $\mu \mathrm{G}$ ) in edge-on and face-on view of the model v70 for selected time-steps: $0.15,0.75$, and 5.00 Gyr. Lower panels: energy density of the cosmic rays and the normalised vectors of the magnetic field at the corresponding time-steps. 
of a positive (blue) and negative (red) azimuthal magnetic field. In the $x z$ cut a similar pattern is revealed but only very close to the disk midplane, that is there are no such structures in the halo. The differential rotation shears the radially aligned structures and forms a spiral pattern of an oppositely directed azimuthal magnetic field, which are clearly visible at $t=0.75 \mathrm{Gyr}$. In the disk at the end of the simulation there is only negatively oriented magnetic azimuthal flux. The magnitude of $B_{\phi}$ is the strongest in the central part of the disk, and it slowly decreases with the radius. The negatively oriented flux is mostly located in the midplane of the galaxy, but it extends also to some vertical height in the form of a disk corona.

The initial state of the cosmic-ray energy follows the density distribution. As the system evolves and the supernovae explode, additional energy fluctuations appear (Fig. 3, bottom panels) . Weak and wide streams of cosmic-ray energy form in the vertical direction; they start from the disk midplane and span up to the top and bottom domain boundaries (see Fig. 3 at $t=0.75$ Gyr). These are the channels that transfer the cosmic-ray energy out of the domain.

The result of each simulation are the $3 \mathrm{D}$ cubes of magnetic field and the CR energy density. Using the CR component as the proxy for the distribution of the relativistic electrons, one can create a synthetic maps of total power and polarised synchrotron radiation (for details see OtmianowskaMazur et al. 2009, Sect. 3). Figure 4 shows the polarisation map for model v70 at $t=5 \mathrm{Gyr}$. The map shows the synthetic distribution of the polarised intensity at $\lambda 6.2 \mathrm{~cm}$ and the polarisation angles, both superimposed onto the column gas density. The polarised intensity in the disk region has a very strong gradient at the edge of the disk. The magnetic-field structure is dominated by the azimuthal component and the vertical slices through the computational domain show that the vertical magnetic-field component is relatively weak. The synthetic polarisation map of the simulated galaxy shows that the magnetic field has a very strong toroidal component and is almost perfectly parallel to the disk plane. Similar results have been found in observation of NGC 1569 by Kepley et al. (2010). This clearly hints at a longterm enhanced SFR in NGC 1569 as the origin of the magnetic field, with the current burst only modulating the field topology. The long enhanced SFR in NGC 1569 is consistent with the results from HST-based colour magnitude analyses (Vallenari \& Bomans 1996).

\section{Conclusions}

We have shown for the first time a global model of the cosmicray-driven dynamo for a dwarf galaxy. The model consists of (1) exploding supernovae that supply the CR energy input and are distributed depending on the local gas density; (2) seeding the dynamo by randomly oriented dipoles injected with the first bursts of supernovae; (3) the Burkert DM profile, and (4) the ISM resistivity. We studied the evolution of the magnetic field for two galaxies characterised by different rotation speeds: $40 \mathrm{~km} \mathrm{~s}^{-1}$ and $70 \mathrm{~km} \mathrm{~s}^{-1}$. We found that

- the cosmic-ray-driven dynamo operating in a dwarf galaxy can amplify regular magnetic fields exponentially in time, up to the saturation level that corresponds to the equipartition magnetic-field strength in real galaxies;

- the $e$-folding time-scales show that fast-rotating objects generate magnetic fields faster than the slow ones, which is consistent with the $\alpha \omega$-dynamo paradigm (Brandenburg \& Subramanian 2005);

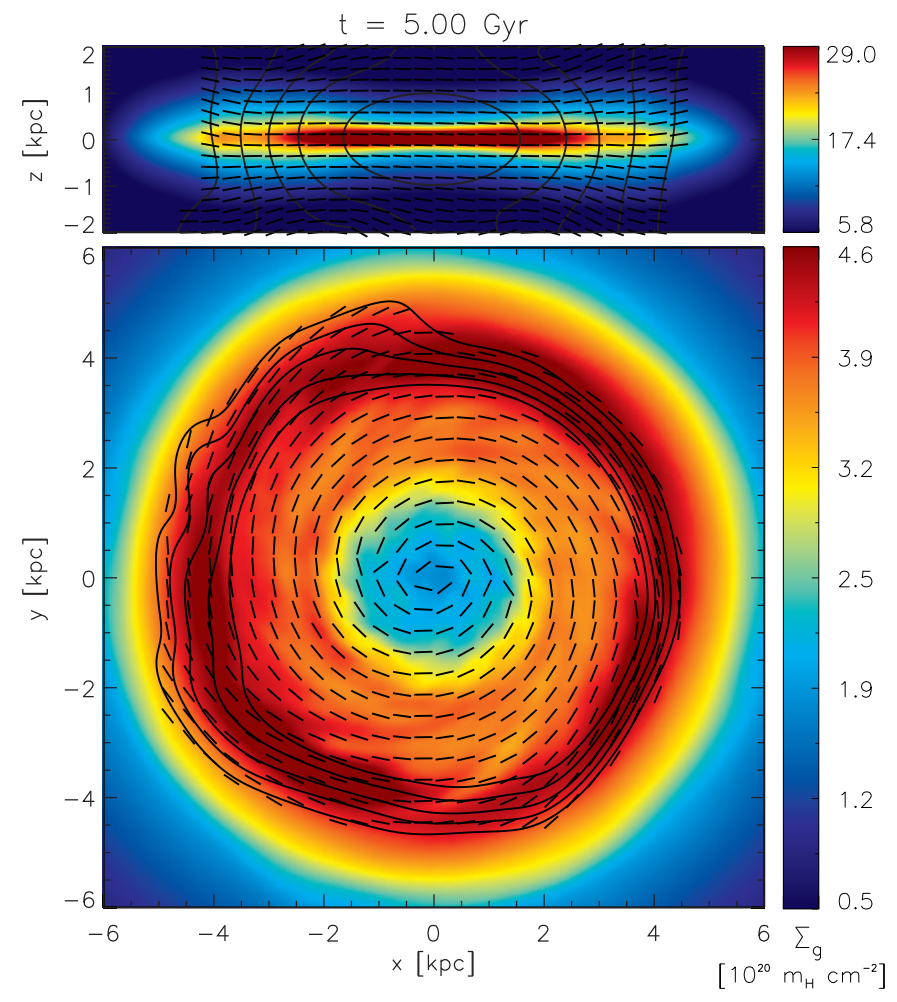

Fig. 4. Polarization map at $\lambda 6.2 \mathrm{~cm}$ for the model v70 at $t=5$ Gyr. The top panel shows the edge-on and the bottom panel the face-on view of the galaxy. The contours of the polarisation intensity and the dashes of the polarisation angles are superimposed onto the column gas density $\left(\Sigma_{\mathrm{g}}\right)$ plot, where $m_{\mathrm{H}}$ is the hydrogen mass. The maps have been smoothed down to the resolution $40^{\prime \prime}$.

- the calculated time-scales for the magnetic-field energy evolution are compatible with those reported by Hanasz et al. (2009b) and Kulpa-Dybeł et al. (2011) for more massive galaxies that show an $e$-folding time of the magnetic-field growth rate of about $300 \mathrm{Myr}$; in our results, the time-scales are about $450 \mathrm{Myr}$ for v70 model and $1000 \mathrm{Myr}$ for v40, implying that the magnetic field in dwarfs is more the result of a long-term slightly enhanced star formation than due to one recent strong burst;

- the $8 \mu \mathrm{G}$ magnetic fields generated in the model v70 are in the range of observed values presented in Chyży et al. (2011). The magnetic field generated in model v40 reaches the saturation phase after about $10 \mathrm{Gyr}$ and the final values are also similar to those of real galaxies. One should keep in mind that the SFR history or interaction with the environment in real galaxies differs from the modelled ones, therefore the magnetic-field values do not match exactly.

The results of our modelling indicate that the cosmic-ray-driven dynamo can explain the observed magnetic fields in dwarf galaxies. In future work we plan to determine the influence of other parameters and perform more simulations to find and reproduce the observed relations.

Acknowledgements. This work was supported by the by the Polish National Science Centre through grants N N203 583440, N N203 511038, and NCN UMO-2011/03/B/ST9/01859. Calculations were made possible thanks to the PLGrid Infrastructure, website: www.plgrid.pl. This research was supported by the partnership program between the Jagellionian University Kraków and the Ruhr-University Bochum. D.J.B. is supported by the DFG special research unit FOR 1254 "Magnetisation of Interstellar and Intergalactic Media: The Prospects of Low-Frequency Radio Observations". We thank J. Gallagher for discussions. 


\section{References}

Beck, R. 2009, Ap\&SS, 320, 77

Bertone, S., Vogt, C., \& Enßlin, T. 2006, MNRAS, 370, 319

Blanton, M. R., Hogg, D. W., \& SDSS Collaboration. 2002, in AAS Meeting Abstracts \#200, BAAS, 34, 679

Booth, C. M., Agertz, O., Kravtsov, A. V., \& Gnedin, N. Y. 2013, ApJ, 777, L16

Boulares, A., \& Cox, D. P. 1990, ApJ, 365, 544

Brandenburg, A., \& Subramanian, K. 2005, Phys. Rep., 417, 1

Burkert, A. 1995, ApJ, 447, L25

Chyży, K. T., Beck, R., Kohle, S., Klein, U., \& Urbanik, M. 2000, A\&A, 355, 128

Chyży, K. T., Knapik, J., Bomans, D. J., et al. 2003, A\&A, 405, 513

Chyży, K. T., Weżgowiec, M., Beck, R., \& Bomans, D. J. 2011, A\&A, 529, A94

Dubois, Y., \& Teyssier, R. 2010, A\&A, 523, A72

Gaensler, B. M., Haverkorn, M., Staveley-Smith, L., et al. 2005, Science, 307, 1610

Giacalone, J., \& Jokipii, J. R. 1999, ApJ, 520, 204

Governato, F., Brook, C., Mayer, L., et al. 2010, Nature, 463, 203

Gressel, O., Elstner, D., Ziegler, U., \& Rüdiger, G. 2008a, A\&A, 486, L35

Gressel, O., Elstner, D., Ziegler, U., \& Rüdiger, G. 2008b, A\&A, 605, L33

Hanasz, M., \& Lesch, H. 2003, A\&A, 412, 331

Hanasz, M., Kowal, G., Otmianowska-Mazur, K., \& Lesch, H. 2004, ApJ,

Hanasz, M., Otmianowska-Mazur, K., Kowal, G., \& Lesch, H. 2006, Astron. Nachr., 327, 469

Hanasz, M., Otmianowska-Mazur, K., Kowal, G., \& Lesch, H. 2009a, A\&A, 498,335

Hanasz, M., Wóltański, D., \& Kowalik, K. 2009b, ApJ, 706, L155
Hanasz, M., Lesch, H., Naab, T., et al. 2013, ApJ, 777, L38

Kennicutt, Jr., R. C. 1989, ApJ, 344, 685

Kepley, A. A., Mühle, S., Everett, J., et al. 2010, ApJ, 712, 536

Klein, U., Wielebinski, R., Haynes, R. F., \& Malin, D. F. 1989, A\&A, 211, 280

Klein, U., Hummel, E., Bomans, D. J., \& Hopp, U. 1996, A\&A, 313, 396

Kowal, G., Lazarian, A., Vishniac, E. T., \& Otmianowska-Mazur, K. 2009, ApJ, 700,63

Kronberg, P. P., Lesch, H., \& Hopp, U. 1999, ApJ, 511, 56

Kulpa-Dybeł, K., Otmianowska-Mazur, K., Kulesza-Żydzik, B., et al. 2011, ApJ, 733, L18

Mac Low, M.-M., \& Ferrara, A. 1999, ApJ, 513, 142

Marcolini, A., Brighenti, F., \& D'Ercole, A. 2003, MNRAS, 345, 1329

Marcolini, A., Brighenti, F., \& D'Ercole, A. 2004, MNRAS, 352, 363

Otmianowska-Mazur, K., Soida, M., Kulesza-Żydzik, B., Hanasz, M., \& Kowal, G. 2009, ApJ, 693, 1

Parker, E. N. 1965, ApJ, 142, 584

Parker, E. N. 1992, ApJ, 401, 137

Ryu, D., Kim, J., Hong, S. S., \& Jones, T. W. 2003, ApJ, 589, 338

Scannapieco, E., \& Brüggen, M. 2010, MNRAS, 405, 1634

Schlickeiser, R., \& Lerche, I. 1985, A\&A, 151, 151

Schmidt, M. 1959, ApJ, 129, 243

Siejkowski, H., Soida, M., Otmianowska-Mazur, K., Hanasz, M., \& Bomans, D. J. 2010, A\&A, 510, A97

Strong, A. W., Moskalenko, I. V., \& Ptuskin, V. S. 2007, Ann. Rev. Nucl. Particle Sci., 57, 285

Vallenari, A., \& Bomans, D. J. 1996, A\&A, 313, 713

Wang, H.-H., Klessen, R. S., Dullemond, C. P., van den Bosch, F. C., \& Fuchs, B. 2010, MNRAS, 407, 705 